\title{
Soft Semi Compactness via Soft Ideals
}

\author{
A. Kandil ${ }^{1}$, O. A. E. Tantawy ${ }^{2}$, S. A. El-Sheikh ${ }^{3}$ and A. M. Abd El-latif $f^{3, *}$ \\ ${ }^{1}$ Mathematics Department, Faculty of Science, Helwan University, Helwan, Egypt \\ ${ }^{2}$ Mathematics Department, Faculty of Science, Zagazig University, Zagazig, Egypt \\ ${ }^{3}$ Mathematics Department, Faculty of Education, Ain Shams University, Cairo, Egypt
}

Received: 27 Aug. 2013, Revised: 24 Nov. 2013, Accepted: 25 Nov. 2013

Published online: 1 Sep. 2014

\begin{abstract}
In the present paper, we have continued to study the properties of soft topological spaces. We introduce new types of soft compactness based on the soft ideal $\tilde{I}$, in a soft topological space $(X, \tau, E)$ namely, soft $\tilde{I}$-compactness, soft semi- $\tilde{I}$-compactness, soft countably-Ĩ-compactness and soft countably semi- $\tilde{I}$-compactness. Also, several of their topological properties are investigated. The behaviour of these concepts under various types of soft functions has obtained.
\end{abstract}

Keywords: Soft set, Soft topological space, Soft interior, Soft closure, Open soft, Closed soft, Soft semi- $\tilde{I}$-compact, Soft S-closed, Soft quasi H-closed, Soft semi lindelöf, Soft countably semi-I-I-compact.

\section{Introduction}

The concept of soft sets was first introduced by Molodtsov [22] in 1999 as a general mathematical tool for dealing with uncertain objects. In [22,23], Molodtsov successfully applied the soft theory in several directions, such as smoothness of functions, game theory, operations research, Riemann integration, Perron integration, probability, theory of measurement, and so on. After presentation of the operations of soft sets [20], the properties and applications of soft set theory have been studied increasingly $[5,16,23,26]$. In recent years, many interesting applications of soft set theory have been expanded by embedding the ideas of fuzzy sets $[2,4,8,18$, $19,20,21,23,24,33]$. To develop soft set theory, the operations of the soft sets are redefined and a uni-int decision making method was constructed by using these new operations [9].

Recently, in 2011, Shabir and Naz [28] initiated the study of soft topological spaces. They defined soft topology on the collection $\tau$ of soft sets over $X$. Consequently, they defined basic notions of soft topological spaces such as open soft and closed soft sets, soft subspace, soft closure, soft nbd of a point, soft separation axioms, soft regular spaces and soft normal spaces and established their several properties. Hussain and Ahmad [27] investigated the properties of open (closed) soft, soft nbd and soft closure. They also defined and discussed the properties of soft interior, soft exterior and soft boundary which are fundamental for further research on soft topology and will strengthen the foundations of the theory of soft topological spaces. Kandil et al.[11] introduced a unification of some types of different kinds of subsets of soft topological spaces using the notions of $\gamma$-operation. Kandil et al.[14] generalize this unification of some types of different kinds of subsets of soft topological spaces using the notions of $\gamma$-operation to supra soft topological spaces. The notion of soft ideal is initiated for the first time by Kandil et al.[12]. They also introduced the concept of soft local function. These concepts are discussed with a view to find new soft topologies from the original one, called soft topological spaces with soft ideal $(X, \tau, E, \tilde{I})$. The concept of generalized closed soft sets in soft topological spaces was introduced by Kannan [15] in 2012. Kandil et al. [13] introduced the concept of supra

*Corresponding author e-mail: Alaa_8560@yahoo.com,dr.Alaa_daby@yahoo.com 
generalized closed soft sets with respect to a soft ideal (supra-II $g$-closed soft for short) in a supra soft topological space $(X, \mu, E)$ and study their properties in detail. Abd El-Monsef et al.[1] studied the relations between ideals and some types of weak compactness. Mahanta and Das in [17] introduced and studied the notions of soft semi-compactness and soft semi-connectedness. So, the main aim of this paper is to study the relation between soft ideals and some types of weak soft compactness. We initiate new types of soft compactness modulo an soft ideal that generalize the concepts, soft semi-compact, soft S-closed, soft semi S-closed, soft semi-lindelöf and other types of soft compactness. Moreover, we study some of their properties and characterizations. This paper, not only can form the theoretical basis for further applications of topology on soft sets, but also lead to the development of information systems.

\section{Preliminaries}

In this section, we present the basic definitions and results of soft set theory which will be needed in the sequel.

Definition 2.1.[22] Let $X$ be an initial universe and $E$ be a set of parameters. Let $P(X)$ denote the power set of $X$ and $A$ be a non-empty subset of $E$. A pair $(F, A)$ denoted by $F_{A}$ is called a soft set over $X$, where $F$ is a mapping given by $F: A \rightarrow P(X)$. In other words, a soft set over $X$ is a parametrized family of subsets of the universe $X$. For a particular $e \in A, F(e)$ may be considered the set of $e$ approximate elements of the soft set $(F, A)$ and if $e \notin A$, then $F(e)=\phi$ i.e

$F_{A}=\{F(e): e \in A \subseteq E, F: A \rightarrow P(X)\}$. The family of all these soft sets over $X$ denoted by $S S(X)_{A}$.

Definition 2.2.[20] Let $F_{A}, G_{B} \in S S(X)_{E}$. Then $F_{A}$ is soft subset of $G_{B}$, denoted by $F_{A} \tilde{\subseteq} G_{B}$, if

(1) $A \subseteq B$, and

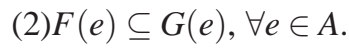

In this case, $F_{A}$ is said to be a soft subset of $G_{B}$ and $G_{B}$ is said to be a soft superset of $F_{A}, G_{B} \tilde{\supseteq} F_{A}$.

Definition 2.3.[20] Two soft subset $F_{A}$ and $G_{B}$ over a common universe set $X$ are said to be soft equal if $F_{A}$ is a soft subset of $G_{B}$ and $G_{B}$ is a soft subset of $F_{A}$.

Definition 2.4.[5] The complement of a soft set $(F, A)$, denoted by $(F, A)^{\prime}$, is defined by $(F, A)^{\prime}=\left(F^{\prime}, A\right)$, $F^{\prime}: A \rightarrow P(X)$ is a mapping given by $F^{\prime}(e)=X-F(e)$, $\forall e \in A$ and $F^{\prime}$ is called the soft complement function of $F$.

Clearly $\left(F^{\prime}\right)^{\prime}$ is the same as $F$ and $\left((F, A)^{\prime}\right)^{\prime}=(F, A)$.
Definition 2.5.[28] The difference between two soft sets $(F, E)$ and $(G, E)$ over the common universe $X$, denoted by $(F, E)-(G, E)$ is the soft set $(H, E)$ where for all $e \in E$, $H(e)=F(e)-G(e)$.

Definition 2.6.[28] Let $(F, E)$ be a soft set over $X$ and $x \in$ $X$. We say that $x \in(F, E)$ read as $x$ belongs to the soft set $(F, E)$ whenever $x \in F(e)$ for all $e \in E$.

Definition 2.7.[20] A soft set $(F, A)$ over $X$ is said to be a NULL soft set denoted by $\tilde{\phi}$ or $\phi_{A}$ if for all $e \in A, F(e)=\phi$ (null set).

Definition 2.8.[20] A soft set $(F, A)$ over $X$ is said to be an absolute soft set denoted by $\tilde{A}$ or $X_{A}$ if for all $e \in A, F(e)=$ $X$. Clearly we have $X_{A}^{\prime}=\phi_{A}$ and $\phi_{A}^{\prime}=X_{A}$.

Definition 2.9.[20] The union of two soft sets $(F, A)$ and $(G, B)$ over the common universe $X$ is the soft set $(H, C)$, where $C=A \cup B$ and for all $e \in C$,

$H(e)=\left\{\begin{array}{l}F(e), e \in A-B, \\ G(e), e \in B-A, \\ F(e) \cup G(e), e \in A \cap B .\end{array}\right.$

Definition 2.10.[20] The intersection of two soft sets $(F, A)$ and $(G, B)$ over the common universe $X$ is the soft set $(H, C)$, where $C=A \cap B$ and for all $e \in C$, $H(e)=F(e) \cap G(e)$. Note that, in order to efficiently discuss, we consider only soft sets $(F, E)$ over a universe $X$ in which all the parameter set $E$ are same. We denote the family of these soft sets by $S S(X)_{E}$.

Definition 2.11.[34] Let $I$ be an arbitrary indexed set and $L=\left\{\left(F_{i}, E\right), i \in I\right\}$ be a subfamily of $S S(X)_{E}$.

(1)The union of $L$ is the soft set $(H, E)$, where $H(e)=\bigcup_{i \in I} F_{i}(e)$ for each $e \in E$. We write $\tilde{U}_{i \in I}\left(F_{i}, E\right)=(H, E)$.

(2)The intersection of $L$ is the soft set $(M, E)$, where $M(e)=\bigcap_{i \in I} F_{i}(e)$ for each $e \in E$. We write $\tilde{\bigcap}_{i \in I}\left(F_{i}, E\right)=(M, E)$.

Definition 2.12.[28] Let $\tau$ be a collection of soft sets over a universe $X$ with a fixed set of parameters $E$, then $\tau \subseteq$ $S S(X)_{E}$ is called a soft topology on $X$ if

(1) $\tilde{X}, \tilde{\phi} \in \tau$, where $\tilde{\phi}(e)=\phi$ and $\tilde{X}(e)=X, \forall e \in E$,

(2)the union of any number of soft sets in $\tau$ belongs to $\tau$,

(3)the intersection of any two soft sets in $\tau$ belongs to $\tau$.

The triplet $(X, \tau, E)$ is called a soft topological space over $X$.

Definition 2.13.[27] Let $(X, \tau, E)$ be a soft topological space. A soft set $(F, A)$ over $X$ is said to be closed soft set in $X$, if its relative complement $(F, A)^{\prime}$ is an open soft set. 
Definition 2.14.[27] $\operatorname{Let}(X, \tau, E)$ be a soft topological space. The members of $\tau$ are said to be open soft sets in $X$. We denote the set of all open soft sets over $X$ by $O S(X, \tau, E)$, or when there can be no confusion by $O S(X)$ and the set of all closed soft sets by $\operatorname{CS}(X, \tau, E)$, or $C S(X)$.

Definition 2.15.[28] Let $(X, \tau, E)$ be a soft topological space and $(F, E) \in S S(X)_{E}$. The soft closure of $(F, E)$, denoted by $\operatorname{cl}(F, E)$ is the intersection of all closed soft super sets of $(F, E)$. Clearly $c l(F, E)$ is the smallest closed soft set over $X$ which contains $(F, E)$ i.e

$\operatorname{cl}(F, E) \quad=\quad \tilde{\cap}\{(H, E)$

$(H, E)$ is closed soft set and $(F, E) \tilde{\simeq}(H, E)\})$.

Definition 2.16.[34] Let $(X, \tau, E)$ be a soft topological space and $(F, E) \in S S(X)_{E}$. The soft interior of $(G, E)$, denoted by $\operatorname{int}(G, E)$ is the union of all open soft subsets of $(G, E)$. Clearly $\operatorname{int}(G, E)$ is the largest open soft set over $X$ which contained in $(G, E)$ i.e

$\operatorname{int}(G, E) \quad=\quad \tilde{\cup}\{(H, E)$

$(H, E)$ is an open soft set and $(H, E) \tilde{\subseteq}(G, E)\})$.

Definition 2.17.[34] The soft set $(F, E) \in S S(X)_{E}$ is called a soft point in $X_{E}$ if there exist $x \in X$ and $e \in E$ such that $F(e)=\{x\}$ and $F\left(e^{\prime}\right)=\phi$ for each $e^{\prime} \in E-\{e\}$, and the soft point $(F, E)$ is denoted by $x_{e}$.

Proposition 2.1.[29] The union of any collection of soft points can be considered as a soft set and every soft set can be expressed as union of all soft points belonging to it.

Definition 2.18.[34] The soft point $x_{e}$ is said to be belonging to the soft set $(G, A)$, denoted by $x_{e} \tilde{\in}(G, A)$, if for the element $e \in A, F(e) \subseteq G(e)$.

Definition 2.19.[34] A soft set $(G, E)$ in a soft topological space $(X, \tau, E)$ is called a soft neighborhood (briefly: nbd) of the soft point $x_{e} \tilde{\in} X_{E}$ if there exists an open soft set $(H, E)$ such that $x_{e} \tilde{\in}(H, E) \tilde{\subseteq}(G, E)$.

A soft set $(G, E)$ in a soft topological space $(X, \tau, E)$ is called a soft neighborhood of the soft $(F, E)$ if there exists an open soft set $(H, E)$ such that $(F, E) \tilde{\in}(H, E) \tilde{\subseteq}(G, E)$. The neighborhood system of a soft point $x_{e}$, denoted by $N_{\tau}\left(x_{e}\right)$, is the family of all its neighborhoods.

Theorem 2.1.[31] Let $(X, \tau, E)$ be a soft topological space. For any soft point $x_{e}, x_{e} \tilde{\in} c l(F, E)$ if and only if each soft neighborhood of $x_{e}$ intersects $(F, E)$.

Definition 2.20.[28] Let $(X, \tau, E)$ be a soft topological space and $Y$ be a non null subset of $X$. Then $\tilde{Y}$ denotes the soft set $(Y, E)$ over $X$ for which $Y(e)=Y \forall e \in E$.

Definition 2.21.[28] Let $(X, \tau, E)$ be a soft topological space, $(F, E) \in S S(X)_{E}$ and $Y$ be a non null subset of $X$. Then the sub soft set of $(F, E)$ over $Y$ denoted by $\left(F_{Y}, E\right)$, is defined as follows:

$$
F_{Y}(e)=Y \cap F(e) \forall e \in E .
$$

In other words $\left(F_{Y}, E\right)=\tilde{Y} \tilde{\cap}(F, E)$.

Definition 2.22.[28] Let $(X, \tau, E)$ be a soft topological space and $Y$ be a non null subset of $X$. Then

$$
\tau_{Y}=\left\{\left(F_{Y}, E\right):(F, E) \in \tau\right\}
$$

is said to be the soft relative topology on $Y$ and $\left(Y, \tau_{Y}, E\right)$ is called a soft subspace of $(X, \tau, E)$.

Theorem 2.2.[28] Let $\left(Y, \tau_{Y}, E\right)$ be a soft subspace of a soft topological space $(X, \tau, E)$ and $(F, E) \in S S(X)_{E}$. Then

(1)If $(F, E)$ is an open soft set in $Y$ and $\tilde{Y} \in \tau$, then $(F, E) \in$ $\tau$.

(2) $(F, E)$ is an open soft set in $Y$ if and only if $(F, E)=$ $\tilde{Y} \tilde{\cap}(G, E)$ for some $(G, E) \in \tau$.

(3) $(F, E)$ is a closed soft set in $Y$ if and only if $(F, E)=$ $\tilde{Y} \tilde{\cap}(H, E)$ for some $(H, E)$ is $\tau$-closed soft set.

Definition 2.23.[11] Let $(X, \tau, E)$ be a soft topological space. A mapping $\gamma: S S(X)_{E} \rightarrow S S(X)_{E}$ is said to be an operation on $O S(X)$ if $F_{E} \tilde{\subseteq} \gamma\left(F_{E}\right) \forall F_{E} \in O S(X)$. The collection of all $\gamma$-open soft sets is denoted by $O S(\gamma)=\left\{F_{E}: F_{E} \tilde{\subseteq} \gamma\left(F_{E}\right), F_{E} \in S S(X)_{E}\right\}$. Also, the complement of $\gamma$-open soft set is called $\gamma$-closed soft set, i.e

$C S(\gamma)=\left\{F_{E}^{\prime}: F_{E}\right.$ is a $\gamma$-opensoft set, $\left.F_{E} \in S S(X)_{E}\right\}$ is the family of all $\gamma$-closed soft sets.

Definition 2.24.[11] Let $(X, \tau, E)$ be a soft topological space. Different cases of $\gamma$-operations on $S S(X)_{E}$ are as follows:

(1)If $\gamma=\operatorname{int}(c l)$, then $\gamma$ is called pre-open soft operator. We denote the set of all pre-open soft sets by $\operatorname{POS}(X, \tau, E)$, or when there can be no confusion by $P O S(X)$ and the set of all pre-closed soft sets by $\operatorname{PCS}(X, \tau, E)$, or $P C S(X)$.

(2)If $\gamma=\operatorname{int}(\operatorname{cl}(i n t))$, then $\gamma$ is called $\alpha$-open soft operator. We denote the set of all $\alpha$-open soft sets by $\alpha O S(X, \tau, E)$, or $\alpha O S(X)$ and the set of all $\alpha$-closed soft sets by $\alpha C S(X, \tau, E)$, or $\alpha C S(X)$.

(3)If $\gamma=c l$ (int), then $\gamma$ is called semi open soft operator. We denote the set of all semi open soft sets by $\operatorname{SOS}(X, \tau, E)$, or $\operatorname{SOS}(X)$ and the set of all semi closed soft sets by $S C S(X, \tau, E)$, or $S C S(X)$.

(4)If $\gamma=c l(\operatorname{int}(c l))$, then $\gamma$ is called $\beta$-open soft operator. We denote the set of all $\beta$-open soft sets by $\beta O S(X, \tau, E)$, or $\beta O S(X)$ and the set of all $\beta$-closed soft sets by $\beta C S(X, \tau, E)$, or $\beta C S(X)$.

Definition 2.25.[11] Let $(X, \tau, E)$ be a soft topological space, $(F, E) \in S S(X)_{E}$ and $x_{e} \in S S(X)_{E}$. Then 
(1) $x_{e}$ is called a $\gamma$ - interior soft point of $(F, E)$ if $\exists$ $(G, E) \in O S(\gamma)$ such that $x_{e} \in(G, E) \tilde{\simeq}(F, E)$, the set of all $\gamma$-interior soft points of $(F, E)$ is called the $\gamma$-soft interior of $(F, E)$ and is denoted by $\gamma-\operatorname{Sint}(F, E) \quad$ consequently, $\quad \gamma-\operatorname{Sint}(F, E)=$ $\tilde{U}\{(G, E):(G, E) \tilde{\subseteq}(F, E),(G, E) \in O S(\gamma)\}$.

(2) $x_{e}$ is called a $\gamma$-cluster soft point of $(F, E)$ if $(F, E) \tilde{\cap}(H, E) \neq \tilde{\phi} \forall(H, E) \in O S(\gamma)$. The set of all $\gamma$-cluster soft points of $(F, E)$ is called $\gamma$-soft closure of $(F, E)$ and is denoted by $\gamma-\operatorname{Scl}(F, E)$ consequently, $\gamma-\operatorname{Scl}(F, E)=\tilde{\bigcap}\{(H, E):(H, E) \in$ $C S(\gamma),(F, E) \tilde{\subseteq}(H, E)\}$.

Definition 2.26. [3] Let $S S(X)_{A}$ and $S S(Y)_{B}$ be families of soft sets, $u: X \rightarrow Y$ and $p: A \rightarrow B$ be mappings. Let $f_{p u}$ : $S S(X)_{A} \rightarrow S S(Y)_{B}$ be a mapping. Then;

(1)If $(F, A) \in S S(X)_{A}$. Then the image of $(F, A)$ under $f_{p u}$, written as $f_{p u}(F, A)=\left(f_{p u}(F), p(A)\right)$, is a soft set in $S S(Y)_{B}$ such that

$f_{p u}(F)(b)$
$\begin{cases}\cup_{a \in p^{-}} 1(b) \cap A & u(F(a)), \quad p^{-1}(b) \cap A \neq \phi, \\ \phi, & \text { otherwise. }\end{cases}$

for all $b \in B$.

(2)If $(G, B) \in S S(Y)_{B}$. Then the inverse image of $(G, B)$ under $f_{p u}$, written as $f_{p u}^{-1}(G, B)=\left(f_{p u}^{-1}(G), p^{-1}(B)\right)$, is a soft set in $S S(X)_{A}$ such that

$f_{p u}^{-1}(G)(a)=\left\{\begin{array}{l}u^{-1}(G(p(a))), \quad p(a) \in B, \\ \phi, \quad \text { otherwise. }\end{array}\right.$

for all $a \in A$.

The soft function $f_{p u}$ is called surjective if $p$ and $u$ are surjective, also is said to be injective if $p$ and $u$ are injective.

Definition 2.27. $[11,17,34]$ Let $\left(X, \tau_{1}, A\right)$ and $\left(Y, \tau_{2}, B\right)$ be soft topological spaces and $f_{p u}: S S(X)_{A} \rightarrow S S(Y)_{B}$ be a function. Then The function $f_{p u}$ is called

(1)Continuous soft if $f_{p u}^{-1}(G, B) \in \tau_{1} \forall(G, B) \in \tau_{2}$.

(2)Open soft if $f_{p u}(G, A) \in \tau_{2} \forall(G, A) \in \tau_{1}$.

(3)Semi open soft if $f_{p u}(G, A) \in \operatorname{SOS}(Y) \forall(G, A) \in \tau_{1}$.

(4)Semi continuous soft function if $f_{p u}^{-1}(G, B) \in \operatorname{SOS}(X) \forall(G, B) \in \tau_{2}$.

(5)Irresolute soft if $f_{p u}^{-1}(G, B) \in \operatorname{SOS}(X) \forall(G, B) \in$ $\operatorname{SOS}(Y)\left[f_{p u}^{-1}(F, B) \in \operatorname{SCS}(X) \forall(F, B) \in S C S(Y)\right]$.

(6)Irresolute open soft (resp. irresolute closed soft) if $f_{p u}(G, A) \in \quad \operatorname{SOS}(Y) \forall \quad(G, A) \quad \in$ $\operatorname{SOS}(X)\left(\right.$ resp. $\left.f_{p u}(F, A) \in S C S(Y) \forall(F, A) \in S C S(Y)\right)$.

Theorem 2.3.[3] Let $S S(X)_{A}$ and $S S(Y)_{B}$ be families of soft sets. For the soft function $f_{p u}: S S(X)_{A} \rightarrow S S(Y)_{B}$, the following statements hold,

(a) $f_{p u}^{-1}\left((G, B)^{\prime}\right)=\left(f_{p u}^{-1}(G, B)\right)^{\prime} \forall(G, B) \in S S(Y)_{B}$. (b) $f_{p u}\left(f_{p u}^{-1}((G, B))\right) \tilde{\subseteq}(G, B) \forall(G, B) \in S S(Y)_{B}$. If $f_{p u}$ is surjective, then the equality holds.

(c) $(F, A) \tilde{\subseteq} f_{p u}^{-1}\left(f_{p u}((F, A))\right) \forall(F, A) \in S S(X)_{A}$. If $f_{p u}$ is injective, then the equality holds.

Definition 2.28.[7] Let $(X, \tau, E)$ be a soft topological space and $x, y \in X$ such that $x \neq y$. Then $(X, \tau, E)$ is called soft Hausdorff space or soft $T_{2}$ space if there exist open soft sets $(F, E)$ and $(G, E)$ such that $x \in(F, E), y \in(G, E)$ and $(F, E) \tilde{\cap}(G, E)=\tilde{\phi}$

Definition 2.29.[10]. A non-empty collection $I$ of subsets of a set $X$ is called an ideal on $X$, if it satisfies the following conditions

(1) $A \in I$ and $B \in I \Rightarrow A \cup B \in I$,

(2) $A \in I$ and $B \subseteq A \Rightarrow B \in I$,

i.e. $I$ is closed under finite unions and subsets.

Definition 2.30.[12] Let $\tilde{I}$ be a non-null collection of soft sets over a universe $X$ with a fixed set of parameters $E$, then $\tilde{I} \subseteq S S(X)_{E}$ is called a soft ideal on $X$ with a fixed set $E$ if

(1) $(F, E) \in \tilde{I}$ and $(G, E) \in \tilde{I} \Rightarrow(F, E) \tilde{\cup}(G, E) \in \tilde{I}$,

(2) $(F, E) \in \tilde{I}$ and $(G, E) \tilde{\subseteq}(F, E) \Rightarrow(G, E) \in \tilde{I}$,

i.e. $\tilde{I}$ is closed under finite soft unions and soft subsets.

Examples 2.1.[12] Let $X$ be a universe set. Then each of the following families is a soft ideal over $X$ with the same set of parameters $E$,

(1) $\tilde{I}=\{\tilde{\phi}\}$,

(2) $\tilde{I}=S S(X)_{E}=\{(F, E):(F, E)$;

is a soft set over $X$ with the fixed set of parameters $E\}$,

(3) $\tilde{I}_{f}=\left\{(F, E) \in S S(X)_{E}:(F, E)\right.$ is finite $\}$, called soft ideal of finite soft sets,

(4) $\tilde{I}_{c}=\left\{(F, E) \in S S(X)_{E}:(F, E)\right.$ is countable $\}$, called soft ideal of countable soft sets,

$(5) \tilde{I}_{(F, E)}=\left\{(G, E) \in S S(X)_{E}:(G, E) \tilde{\subseteq}(F, E)\right\}$.

(6) $\tilde{I}_{n}=\left\{(G, E) \in S S(X)_{E}: \operatorname{int}(\operatorname{cl}(G, E))=\tilde{\phi}\right\}$, called soft ideal of nowhere dense soft sets in $(X, \tau, E)$.

Definition 2.31.[34] A family $\Psi$ of soft sets is called a soft cover of a soft set $(F, E)$ if

$$
(F, E) \tilde{\simeq} \tilde{\cup}\left\{\left(F_{i}, E\right):\left(F_{i}, E\right) \in \Psi, i \in I\right\} .
$$

It is an open soft cover if each member of $\Psi$ is an open soft set. A soft subcover of $\Psi$ is a subfamily of $\Psi$ which is also a soft cover of $(F, E)$.

Definition 2.32.[34] A family $\Psi$ of soft sets is said to be have the finite intersection property (FIP for short) if the soft intersection of the members of each finite subfamily of $\Psi$ is not null soft set. 
Definition 2.33.[34] A soft topological space $(X, \tau, E)$ is called soft compact space if each open soft cover of $\tilde{X}$ has a finite soft subcover.

Definition 2.34.[31] A soft topological space $(X, \tau, E)$ is called soft lindelöf if each open soft cover of $\tilde{X}$ has a soft countable subcover.

Definition 2.35.[17] A soft topological space $(X, \tau, E)$ is called soft semi compact space if each soft cover by semi open soft sets of $\tilde{X}$ has a finite soft subcover.

\section{Soft semi- $\tilde{I}$-compact spaces}

Definition 3.1. A soft subset $(F, E)$ of the space $(X, \tau, E, \tilde{I})$ is said to be soft $\tilde{I}$-compact if every soft cover $\left\{\left(G_{\alpha}, E\right)\right.$ : $\alpha \in \Lambda\}$ of $(F, E)$ by open soft sets, there exists a finite subset $\Lambda_{o}$ of $\Lambda$ such that $(F, E) \backslash \tilde{\cup}_{\alpha \in \Lambda_{o}}\left(G_{\alpha}, E\right) \in \tilde{I}$. The space $(X, \tau, E, \tilde{I})$ is said to be soft $\tilde{I}$-compact if $\tilde{X}$ is soft $\tilde{I}$-compact as a soft subset.

\section{Proposition 3.1.}

(1)Every soft compact soft topological space $(X, \tau, E)$ is soft $\tilde{I}$ - compact for any soft ideal $\tilde{I}$ on $\tilde{X}$.

(2)If $\tilde{I}=\{\tilde{\phi}\}$, then $(X, \tau, E)$ is soft compact $\Leftrightarrow$ it is soft $\tilde{I}$-compact.

(3)If the $\star$-soft topology $\left(X, \tau^{*}, E\right)$ is soft $\tilde{I}$-compact, then the soft topology $(X, \tau, E)$ is soft $\tilde{I}$-compact.

Proof. Immediate.

Theorem 3.1. Let $(X, \tau, E)$ be a soft topological space, $\tilde{I}$ be a soft ideal on $X$ with the same set of parameters $E$ and $\left(X, \tau^{*}, E\right)$ be its $\star$-soft topological space. Then $(X, \tau, E)$ is soft $\tilde{I}$-compact if and only if $\left(X, \tau^{*}, E\right)$ is soft $\tilde{I}$-compact.

Proof. Let $(X, \tau, E)$ be a soft $\tilde{I}$-compact and $\left\{\left(G_{\alpha}, E\right): \alpha \in \Lambda\right\}$ be $\tau^{*}$-open soft cover of $\tilde{X}$. Then $\left(G_{\alpha}, E\right)=\left(V_{\alpha}, E\right)-\left(I_{\alpha}, E\right) \forall \alpha \in \Lambda$, where $\left(V_{\alpha}, E\right) \in \tau$ and $\left(I_{\alpha}, E\right) \in \tilde{I}$. It follows that $\left\{\left(V_{\alpha}, E\right): \alpha \in \Lambda\right\}$ is a $\tau$-open soft cover of $\tilde{X}$. Thus by soft $\tilde{I}$-compactness of $(X, \tau, E), \exists \Lambda_{o} \subseteq \Lambda$ finite such that $\tilde{X}-\tilde{\cup}_{\alpha \in \Lambda_{o}}\left(V_{\alpha}, E\right) \in \tilde{I}$. So $\tilde{X}-\tilde{\cup}_{\alpha \in \Lambda_{o}}\left(G_{\alpha}, E\right)=\tilde{X}-\tilde{\cup}_{\alpha \in \Lambda_{o}}\left[\left(V_{\alpha}, E\right)-\right.$ $\left.\left(I_{\alpha}, E\right)\right] \tilde{\subseteq}\left[\tilde{X}-\tilde{\cup}_{\alpha \in \Lambda_{o}}\left(V_{\alpha}, E\right)\right] \tilde{\cup}\left[\tilde{\cup}_{\alpha \in \Lambda_{o}}\left(I_{\alpha}, E\right)\right] \in \tilde{I}$, where $\left(I_{\alpha}, E\right) \in \tilde{I} \forall \alpha \in \Lambda$. Hence $\left(X, \tau^{*}, E\right)$ is soft $\tilde{I}$-compact. The necessary of the theorem follows from Proposition 3.1 (3).

Theorem 3.2. Let $(X, \tau, E)$ be a soft topological space, $\tilde{I}$ be a soft ideal on $X$ with the same set of parameters $E$ and $\left(X, \tau^{*}, E\right)$ be its $\star$-soft topological space. Then the following implications hold.

$$
\begin{gathered}
\left(X, \tau^{*}, E\right) \text { is soft compact } \Rightarrow(X, \tau, E) \text { is soft compact } \\
\Downarrow
\end{gathered}
$$

$\left(X, \tau^{*}, E\right)$ is soft $\tilde{I}$-compact $\Leftrightarrow(X, \tau, E)$ is soft $\tilde{I}$-compact

Proof. Immediate from Proposition 3.1 and Theorem 3.1 .

Definition 3.2. A family $\Psi$ of semi open soft sets is called a semi open soft cover of a soft set $(F, E)$ if

$$
(F, E) \tilde{\simeq} \tilde{\cup}\left\{\left(F_{i}, E\right):\left(F_{i}, E\right) \in \Psi, i \in I\right\} .
$$

A semi open soft subcover of $\Psi$ is a subfamily of $\Psi$ which is also a semi open soft cover of $(F, E)$.

Definition 3.3.A soft subset $(F, E)$ of the space $(X, \tau, E, \tilde{I})$ is said to be soft semi- $\tilde{I}$-compact if for every semi open cover $\left\{\left(G_{\alpha}, E\right): \alpha \in \Lambda\right\}$ of $(F, E)$ there exists a finite subset $\Lambda_{o}$ of $\Lambda$ such that

$$
(F, E)-\tilde{\cup}_{\alpha \in \Lambda_{o}}\left(G_{\alpha}, E\right) \in \tilde{I} .
$$

The space $(X, \tau, E, \tilde{I})$ is said to be soft semi- $\tilde{I}$-compact if $\tilde{X}$ is soft semi- $\tilde{I}$-compact as a soft subset.

Proposition 3.2. Let $(X, \tau, E, \tilde{I})$ be a soft topological space with soft ideal with the same set of parameters $E$ and $\left(X, \tau^{*}, E\right)$ its $\star$-soft topology. Then:

(1)If $(X, \tau, E, \tilde{I})$ is a soft semi- $\tilde{I}$-compact, then it is soft $\tilde{I}$ compact.

(2)If $\left(X, \tau^{*}, E\right)$ is a soft semi- $\tilde{I}$-compact, then $(X, \tau, E)$ is also soft semi-compact.

Proof. Immediate.

Theorem 3.3. A soft topological space $(X, \tau, E)$ is soft semi compact if and only if $\left(X, \tau, E, \tilde{I}_{f}\right)$ is soft semi- $\tilde{I}_{f}$-compact, where $\tilde{I}_{f}$ is the soft ideal of finite soft subsets of $\tilde{X}$.

Proof. Let $(X, \tau, E)$ be a soft semi compact topological space and let $\left\{\left(G_{\alpha}, E\right): \alpha \in \Lambda\right\}$ be a semi open soft cover of $\tilde{X}$. Then there exists a finite subset $\Lambda_{o}$ of $\Lambda$ such that $\tilde{X}=\tilde{\cup}_{\alpha \in \Lambda_{o}}\left(G_{\alpha}, E\right)$. It follows that $\tilde{X}-\tilde{U}_{\alpha \in \Lambda_{o}}\left(G_{\alpha}, E\right)=\tilde{\phi} \in \tilde{I}_{f}$. Hence $\left(X, \tau, E, \tilde{I}_{f}\right)$ is soft semi- $\tilde{I}_{f}$-compact.

Conversely, let $\left(X, \tau, E, \tilde{I}_{f}\right)$ be a soft semi- $\tilde{I}_{f}$-compact and let $\left\{\left(G_{\alpha}, E\right): \alpha \in \Lambda\right\}$ be a semi open soft cover of $\tilde{X}$. Then there exists a finite subset $\Lambda_{o}$ of $\Lambda$ such that $\tilde{X}-\tilde{\cup}_{\alpha \in \Lambda_{o}}\left(G_{\alpha}, E\right) \in \tilde{I}_{f}$. Thus $\tilde{X}=\tilde{\cup}_{\alpha \in \Lambda_{o}}\left(G_{\alpha}, E\right)$. Hence $(X, \tau, E)$ is soft semi compact.

Theorem 3.4. Let $(X, \tau, E, \tilde{I})$ be a soft semi- $\tilde{I}$-compact space and $\tilde{J}$ be a soft ideal on $X$ with the same set of parameters $E$ such that $\tilde{I} \subseteq \tilde{J}$. Then $(X, \tau, E, \tilde{J})$ is a soft semi-J-compact.

Proof. Immediate.

Definition 3.4. A soft subset $(F, E)$ of the soft topological space $(X, \tau, E)$ is said to be soft $\mathrm{S}$-closed if each semi open 
soft cover of $(F, E)$ has a finite soft subcover whose soft closure covers $(F, E)$. The space $(X, \tau, E)$ is said to be soft $\mathrm{S}$-closed if $\tilde{X}$ is soft $\mathrm{S}$-closed as a soft subset.

Definition 3.5.A soft subset $(F, E)$ of the soft topological space $(X, \tau, E)$ is said to be soft semi S-closed if each semi open soft cover of $(F, E)$ has a finite semi open soft subcover whose semi soft closure covers $(F, E)$. The space $(X, \tau, E)$ is said to be semi S-closed soft if $\tilde{X}$ is a semi S-closed soft as a soft subset.

Definition 3.6. A soft subset $(F, E)$ of the soft topological space $(X, \tau, E)$ is said to be soft Quasi H-closed (soft QHC for short) if each open soft cover of $(F, E)$ has a finite semi open soft subcover whose soft closure covers $(F, E)$. The space $(X, \tau, E)$ is said to be soft $\mathrm{QHC}$ if $\tilde{X}$ is soft $\mathrm{QHC}$ as a soft subset.

Theorem 3.5. If the space $\left(X, \tau, E, \tilde{I}_{f}\right)$ is soft semi- $\tilde{I}_{f}$-compact, then $(X, \tau, E)$ is soft S-closed (resp. soft semi S-closed, soft QHC).

Proof. We give the proof for the case of soft S-closed. Let $\left\{\left(G_{\alpha}, E\right): \alpha \in \Lambda\right\}$ be a semi open soft cover of $\tilde{X}$. Then there exists a finite subset $\Lambda_{o}$ of $\Lambda$ such that $\tilde{X}-\tilde{\cup}_{\alpha \in \Lambda_{o}}\left(G_{\alpha}, E\right) \in \tilde{I}_{f}$. This means that $\tilde{X}=\tilde{\cup}_{\alpha \in \Lambda_{o}}\left(G_{\alpha}, E\right) \subseteq \tilde{\cup}_{\alpha \in \Lambda_{o}} \operatorname{cl}\left(G_{\alpha}, E\right)$. Hence $\tilde{X}=\tilde{\cup}_{\alpha \in \Lambda_{o}} \operatorname{cl}\left(G_{\alpha}, E\right)$. Therefore $(X, \tau, E)$ is soft S-closed soft. The rest of the proof is similar.

Theorem 3.6. Let $(X, \tau, E, \tilde{I})$ be a soft topological space with soft ideal. If $\tilde{I}_{n} \subseteq \tilde{I}$ and $(X, \tau, E)$ is soft S-closed, then $(X, \tau, E)$ is soft semi- $\tilde{I}$-compact.

Proof. Let $\left\{\left(G_{\alpha}, E\right): \alpha \in \Lambda\right\}$ be a semi open soft cover of $\tilde{X}$. Then there exists a finite subset $\Lambda_{o}$ of $\Lambda$ such that $\tilde{X}=\tilde{\cup}_{\alpha \in \Lambda_{o}} c l\left(G_{\alpha}, E\right) \tilde{\widetilde{\subseteq}} \tilde{\cup}_{\alpha \in \Lambda_{o}} \operatorname{cl}\left(\operatorname{int}\left(G_{\alpha}, E\right)\right)$, where $\left(G_{\alpha}, E\right) \in \operatorname{SOS}(X)$. It follows that $\tilde{X}=\tilde{\cup}_{\alpha \in \Lambda_{o}} \operatorname{cl}\left(\operatorname{int}\left(G_{\alpha}, E\right)\right)$. Hence $\tilde{X}-$ $\tilde{\cup}_{\alpha \in \Lambda_{o}} \operatorname{cl}\left(\operatorname{int}\left(G_{\alpha}, E\right)\right) \tilde{\subseteq} \tilde{X} \tilde{\cap} \operatorname{int}\left(\operatorname{cl}\left(\tilde{\cup}_{\alpha \in \Lambda_{o}}\left(G_{\alpha}, E\right)\right)^{\prime}\right) \tilde{\tilde{X}} \operatorname{int}(\operatorname{cl}(\tilde{X}$ $\left.\left.\tilde{\cup}_{\alpha \in \Lambda_{o}} \operatorname{cl}\left(G_{\alpha}, E\right)\right)\right) \quad=\quad \tilde{\phi}$. Thus $\tilde{X}-\tilde{\cup}_{\alpha \in \Lambda_{o}} c l\left(G_{\alpha}, E\right) \in \tilde{I}_{n} \subseteq \tilde{I}$. Therefore, $(X, \tau, E)$ is soft semi- $\tilde{I}$-compact.

\section{Theorem 3.7.}

Let $(X, \tau, E)$ be a soft topological space. Then $(X, \tau, E)$ is soft semi- $\tilde{I}_{n}$-compact if and only if $(X, \tau, E)$ is soft $\mathrm{S}$ closed.

Proof. Let $\left\{\left(G_{\alpha}, E\right): \alpha \in \Lambda\right\}$ be a semi open soft cover of $\tilde{X}$. Then there exists a finite subset $\Lambda_{o}$ of $\Lambda$ such that $\tilde{X}-\tilde{\cup}_{\alpha \in \Lambda_{o}}\left(G_{\alpha}, E\right) \in \tilde{I}_{n}$ which implies $\tilde{\phi}=\operatorname{int}\left(\operatorname{cl}\left(\tilde{X}-\tilde{\cup}_{\alpha \in \Lambda_{o}}\left(G_{\alpha}, E\right)\right)\right)=$ $\operatorname{int}\left(\operatorname{cl}\left(\tilde{X} \tilde{\cap}\left[\tilde{\cap}_{\alpha \in \Lambda_{o}}\left(G_{\alpha}, E\right)^{\prime}\right)\right]\right) \supseteq \operatorname{Dint}\left(\tilde{X} \tilde{\cap}\left[\tilde{\cap} \in_{\alpha \in \Lambda_{o}} \operatorname{int}\left(\operatorname{cl}\left(G_{\alpha}, E\right)^{\prime}\right)\right]\right.$ $\tilde{X} \tilde{\cap}\left[\tilde{\cup}_{\alpha \in \Lambda_{o}} \operatorname{int}\left(\operatorname{cl}\left(G_{\alpha}, E\right)^{\prime}\right)\right]$ $\tilde{X} \tilde{\cap}\left[\tilde{\cap}_{\alpha \in \Lambda_{o}} \operatorname{int}\left(\operatorname{cl}\left(G_{\alpha}, E\right)^{\prime}\right)\right]=\tilde{X}-\left(\tilde{\cup}_{\alpha \in \Lambda_{o}} \operatorname{cl}\left(\operatorname{int}\left(G_{\alpha}, E\right)\right)\right)$, where $(G, E)^{\prime} S C S(X)$
$\tilde{X}=\tilde{\cup}_{\alpha \in \Lambda_{o}} \operatorname{cl}\left(\operatorname{int}\left(\left(G_{\alpha}, E\right)\right)\right) \tilde{\subseteq} \tilde{\cup}_{\alpha \in \Lambda_{o}} \operatorname{cl}\left(\left(G_{\alpha}, E\right)\right)$. It follows that $\tilde{X}=\tilde{\cup}_{\alpha \in \Lambda_{o}} \operatorname{cl}\left(\left(G_{\alpha}, E\right)\right)$. Thus $(X, \tau, E)$ is soft S-closed.

Conversely, Let $\left\{\left(G_{\alpha}, E\right): \alpha \in \Lambda\right\}$ be a semi open soft cover of $\tilde{X}$. Then there exists a finite subset $\Lambda_{o}$ of $\Lambda$ such that $\quad \tilde{X}=\tilde{\cup}_{\alpha \in \Lambda_{o}} \operatorname{cl}\left(\left(G_{\alpha}, E\right)\right)$. So $\tilde{X}-\tilde{\cup}_{\alpha \in \Lambda_{o}} \operatorname{cl}\left(\left(G_{\alpha}, E\right)\right)=\tilde{X} \tilde{\cap}\left[\tilde{\cap}_{\alpha \in \Lambda_{o}} \operatorname{int}\left(G_{\alpha}, E\right)^{\prime}\right]=\tilde{\phi}$. Since $\quad\left(G_{\alpha}, E\right)^{\prime} \in \operatorname{SCS}(X)$. Then $\tilde{X} \quad-\quad \tilde{U}_{\alpha \in \Lambda_{o}} \operatorname{cl}\left(\operatorname{int}\left(G_{\alpha}, E\right)\right)=$ $\tilde{X} \tilde{\cap}\left[\tilde{\cap}_{\alpha \in \Lambda_{o}} \operatorname{int}\left(\operatorname{int}\left(\operatorname{cl}\left(G_{\alpha}, E\right)^{\prime}\right)\right)\right] \tilde{\subseteq} \tilde{X} \tilde{\cap}\left[\tilde{\cap}{ }_{\alpha \in \Lambda_{o}} \operatorname{int}\left(G_{\alpha}, E\right)^{\prime}\right)=$ $\tilde{\phi}$. This implies that $\tilde{\phi}=\operatorname{int}\left(\operatorname{cl}\left(\tilde{X}-\tilde{\cup}_{\alpha \in \Lambda_{o}} \operatorname{cl}\left(\operatorname{int}\left(G_{\alpha}, E\right)\right)\right)\right)=$ $\operatorname{int}\left(\operatorname{cl}\left(\tilde{X} \tilde{\cap}\left[\tilde{\cap}_{\alpha \in \Lambda_{o}} \operatorname{int}\left(\operatorname{cl}\left(G_{\alpha}, E\right)^{\prime}\right)\right]\right)\right) \supseteq \operatorname{int}(\operatorname{cl}(\tilde{X}$ $\left.\left.\tilde{\cup}_{\alpha \in \Lambda_{o}}\left(G_{\alpha}, E\right)\right)\right)$. It follows that $\tilde{X}-\tilde{\cup}_{\alpha \in \Lambda_{o}}\left(G_{\alpha}, E\right) \in \tilde{I}_{n}$. Thus $(X, \tau, E)$ is soft semi- $\tilde{I}_{n}$-compact which completes the proof.

Definition 3.7. A soft subset $(F, E)$ of the topological space $(X, \tau, E)$ is soft semi lindelöf if each semi open soft cover of $\tilde{X}$ has a soft countable subcover.

Theorem 3.8. If the space $\left(X, \tau, E, \tilde{I}_{c}\right)$ is soft semi- $\tilde{I}_{c}$-compact, then the space $(X, \tau, E)$ is soft semi lindelöf, where $\tilde{I}_{c}$ is the soft ideal of countable soft subsets of $\tilde{X}$.

Proof. Let $\left\{\left(G_{\alpha}, E\right): \alpha \in \Lambda\right\}$ be a semi open soft cover of $\tilde{X}$. Then there exists a finite subset $\Lambda_{o}$ of $\Lambda$ such that $\tilde{X}-\tilde{\cup}_{\alpha \in \Lambda_{o}}\left(G_{\alpha}, E\right) \in \tilde{I}_{c}$. Since $\tilde{X}=$ $\tilde{\cup}\left[\tilde{X}-\tilde{\cup}_{\alpha \in \Lambda_{o}}\left(G_{\alpha}, E\right)\right]=\tilde{\cap}\left[\tilde{X} \tilde{\cup}\left(\tilde{\cap}_{\alpha \in \Lambda_{o}}\left(G_{\alpha}, E\right)\right)^{\prime}\right]=\tilde{X}$. This means that $\tilde{X}-\tilde{\cup}_{\alpha \in \Lambda_{o}}\left(G_{\alpha}, E\right)$ is a soft countable subcover of $\tilde{X}$. Therefore $(X, \tau, E)$ is soft semi lindelöf.

Corollary 3.1. If the space $\left(X, \tau, E, \tilde{I}_{c}\right)$ is soft semi- $\tilde{I}_{C}$-compact, then the space $(X, \tau, E)$ is soft lindelöf.

Proof. Immediate from Theorem 3.8.

Theorem 3.9. Let $\left(X_{1}, \tau_{1}, A, \tilde{I}\right)$ be a soft topological space - with soft ideal, $\left(X_{2}, \tau_{2}, B\right)$ be a soft topological space and $f_{p u}:\left(X_{1}, \tau_{1}, A, \tilde{I}\right) \rightarrow\left(X_{2}, \tau_{2}, B\right)$ be a soft function. Then $f_{p u}(\tilde{I})=\left\{f_{p u}((F, A)):(F, A) \in \tilde{I}\right\}$ is a soft ideal on $X_{2}$.

Proof. Obvious.

Theorem 3.10. Let $\left(X_{1}, \tau_{1}, A, \tilde{I}\right)$ be a soft topological space with soft ideal, $\left(X_{2}, \tau_{2}, B\right)$ be a soft topological space and $f_{p u}:\left(X_{1}, \tau_{1}, A, \tilde{I}\right) \rightarrow\left(X_{2}, \tau_{2}, B\right)$ be an irresolute surjective soft function. If $\left(X_{1}, \tau_{1}, A\right)$ is soft semi- $\tilde{I}$ - compact, then $\left(X_{2}, \tau_{2}, B\right)$ is soft semi- $f_{p u}(\tilde{I})$-compact.

Proof. Let $\left\{\left(G_{\alpha}, B\right): \alpha \in \Lambda\right\}$ be a $\tau_{2}$-semi open soft cover of $\tilde{X}_{2}$. Since $f_{p u}$ is an irresolute soft function, then $\left\{f_{p u}^{-1}\left(G_{\alpha}, B\right): \alpha \in \Lambda\right\}$ is a $\tau_{1}$-semi open soft cover of $\tilde{X}_{1}$. By hypothesis, there exists a finite subset $\Lambda_{o}$ of $\Lambda$ such that $\tilde{X}_{1}-\tilde{\cup}\left\{f_{p u}^{-1}\left(G_{\alpha}, B\right): \alpha \in \Lambda_{o}\right\} \in \tilde{I}$. This implies that $f_{p u}\left[\tilde{X}_{1}-\tilde{\cup}\left\{f_{p u}^{-1}\left(G_{\alpha}, B\right): \alpha \in \Lambda_{o}\right\}\right]=$ $\tilde{X}_{2}-\tilde{\cup}_{\alpha \in \Lambda_{o}}\left(G_{\alpha}, B\right) \in f_{p u}(\tilde{I})$ from Theorem 2.3. Therefore 
$\left(X_{2}, \tau_{2}, B\right)$ is soft semi- $f_{p u}(\tilde{I})$-compact.

The following theorem gives the sufficient condition for the inverse image of a soft ideal to be a soft ideal.

Theorem 3.11. Let $\left(X_{1}, \tau_{1}, A, \quad\left(X_{2}, \tau_{2}, B\right)\right.$ be soft topological spaces, $f_{p u}:\left(X_{1}, \tau_{1}, A,\right) \rightarrow\left(X_{2}, \tau_{2}, B\right)$ be an injective soft function and $\tilde{J}$ be a soft ideal on $X_{2}$. Then $f_{p u}^{-1}(\widetilde{J})$ is a soft ideal on $X_{1}$.

Proof. Obvious.

Theorem 3.12. Let $\left(X_{1}, \tau_{1}, A\right)$ be a soft topological space, $\left(X_{2}, \tau_{2}, B, \tilde{J}\right)$ be a soft topological space with soft ideal and $f_{p u}:\left(X_{1}, \tau_{1}, A\right) \rightarrow\left(X_{2}, \tau_{2}, B, \widetilde{J}\right)$ be a bijection irresolute open soft function. If $\left(X_{2}, \tau_{2}, B\right)$ is soft semi- $\tilde{J}$-compact, then $\left(X_{1}, \tau_{1}, A\right)$ is soft semi- $f_{p u}^{-1}(\tilde{J})$-compact.

Proof. Let $\left\{\left(G_{\alpha}, A\right): \alpha \in \Lambda\right\}$ be a $\tau_{1}$-semi open soft cover of $\tilde{X}_{1}$. Since $f_{p u}$ is a bijection irresolute open soft function, then $\left\{f_{p u}\left(G_{\alpha}, A\right): \alpha \in \Lambda\right\}$ is a $\tau_{2}$-semi open soft cover of $\tilde{X}_{2}$. By hypothesis, there exists a finite subset $\Lambda_{o}$ of $\Lambda$ such that $\tilde{X}_{2}-\tilde{\cup}\left\{f_{p u}\left(G_{\alpha}, A\right): \alpha \in \Lambda_{o}\right\} \in \tilde{I}$. This implies that $f_{p u}^{-1}\left[\tilde{X}_{2}-\tilde{\cup}\left\{f_{p u}\left(G_{\alpha}, A\right): \alpha \in \Lambda_{o}\right\}\right]=$ $\tilde{X}_{1}-\tilde{\cup}_{\alpha \in \Lambda_{o}}\left(G_{\alpha}, A\right) \in f_{p u}^{-1}(\tilde{J})$ from Theorem 2. Therefore $\left(X_{1}, \tau_{1}, A\right)$ is soft semi- $f_{p u}^{-1}(\tilde{J})$-compact.

Theorem 3.13. Let $(X, \tau, E, \tilde{I})$ be a soft topological space with soft ideal. Then the following are equivalent:

(1) $(X, \tau, E)$ is soft semi- $\tilde{I}$-compact.

(2)For every family $\left\{\left(F_{j}, E\right): j \in J\right\}$ of semi closed soft subsets of $\tilde{X}$ for which $\tilde{\cap}\left\{\left(F_{j}, E\right): j \in J\right\}=\tilde{\phi}$, there exists finite $J_{o} \subseteq J$ such that $\tilde{\cap}\left\{\left(F_{j}, E\right): j \in J_{o}\right\} \in \tilde{I}$.

Proof.

$(1) \Rightarrow(2)\left\{\left(F_{j}, E\right): j \in J\right\}$ be a family of semi closed soft sets of $\tilde{X}$ such that $\tilde{\cap}\left\{\left(F_{j}, E\right): j \in J_{o}\right\}=\tilde{\phi}$. Then $\left\{\tilde{X}-\left(F_{j}, E\right): j \in J\right\}$ is a family of semi open soft sets of $\tilde{X}$ such that $\tilde{X}=\tilde{U}\left\{\tilde{X}-\left(F_{j}, E\right): j \in J\right\}$. By (1), there exists finite $J_{o} \subseteq J$ such that $\tilde{X}-\left(\tilde{\cup}\left\{\tilde{X}-\left(F_{j}, E\right): j \in J_{o}\right\}\right) \in \tilde{I}$, i.e $\tilde{X}-\left(\tilde{X} \tilde{\cup}\left(\tilde{\cap}_{j \in J_{o}}\left(F_{j}, E\right)^{\prime}\right)=\tilde{X}-\left(\tilde{X} \tilde{\cap}\left(\tilde{\cup}_{j \in J_{o}}\left(F_{j}, E\right)^{\prime}\right)=\right.\right.$ $\tilde{X}-\left(\tilde{X} \tilde{\cap}\left(\tilde{\cap}_{j \in J_{o}}\left(F_{j}, E\right)\right)^{\prime}\right)=\tilde{X}-\left(\tilde{X}-\tilde{\cap}_{j \in J_{o}}\left(F_{j}, E\right)\right)=$ $\tilde{\cap}_{j \in J_{o}}\left(F_{j}, E\right) \in \tilde{I}$.

(2) $\Rightarrow(1)$ Suppose that $\left\{\left(G_{j}, E\right): j \in J\right\}$ be a family of semi open soft cover of $\tilde{X}$. Then $\left\{\tilde{X}-\left(G_{j}, E\right): j \in J\right\}$ is a family of semi closed soft sets of $\tilde{X}$ with $\tilde{\cap}_{j \in J}(\tilde{X}-$ $\left.\left(G_{j}, E\right)\right)=\tilde{\phi}$. By (2), there exists finite $J_{o} \subseteq J$ such that $\tilde{\cap}_{j \in J}\left(\tilde{X}-\left(G_{j}, E\right)\right) \in \tilde{I}$. Thus $\tilde{\cap}_{j \in J_{o}}\left(\tilde{X}-\left(G_{j}, E\right)\right)=$ $\tilde{X}-\tilde{\cup}_{j \in J_{o}}\left(G_{j}, E\right) \in \tilde{I}$. Therefore, $(X, \tau, E)$ is soft semi$\tilde{I}$-compact.

Remark 3.1. If $\tilde{I}=\tilde{\phi}$ in the previous theorem, we obtain the standard characterizations of soft semi-compactness which mentioned in [17].
Definition 3.8. Let $(X, \tau, E)$ be a soft topological space and $(F, E) \in S S(X)_{E}$. Then $(F, E)$ is called regular open soft set (resp. regular closed soft set) if $(F, E)=\operatorname{int}(\operatorname{cl}(F, E))($ resp. $(F, E)=\operatorname{cl}(\operatorname{int}(F, E)))$. We denote the set of all regular open soft sets by $\operatorname{ROS}(X, \tau, E)$, or when there can be no confusion by $R O S(X)$ and the set of all regular closed soft sets by $R C S(X, \tau, E)$, or $R C S(X)$.

Theorem 3.14. If $(X, \tau, E, \tilde{I})$ soft semi-I-I-compact, then for every cover $\left\{\left(F_{j}, E\right): j \in J\right\}$ of regular closed soft subsets of $\tilde{X}$, there exists finite $J_{o} \subseteq J$ such that $\tilde{X}-\tilde{\cup}\left\{\left(F_{j}, E\right): j \in\right.$ $\left.J_{o}\right\} \in \tilde{I}$.

Proof. Let $\left\{\left(F_{j}, E\right): j \in J\right\}$ be a cover of regular closed soft sets of $\tilde{X}$. Since every regular closed soft set is semi open soft. Then $\left\{\left(F_{j}, E\right): j \in J\right\}$ is a cover of semi open soft sets of $\tilde{X}$. By hypothesis, there exists finite $J_{o} \subseteq J$ such that $\tilde{X}-\tilde{\cup}\left\{\left(F_{j}, E\right): j \in J_{o}\right\} \in \tilde{I}$.

Theorem 3.15. Let $(X, \tau, E, \tilde{I})$ be a soft topological space with soft ideal. If $(X, \tau, E)$ is soft semi- $\tilde{I}$-compact, then for every pre open soft cover $\left\{\left(F_{j}, E\right): j \in J\right\}$ of $\tilde{X}$, there exists finite $J_{o} \subseteq J$ such that $\tilde{X}-\tilde{\cup}\left\{c l\left(F_{j}, E\right): j \in J_{o}\right\} \in \tilde{I}$.

Proof. Let $\left\{\left(F_{j}, E\right): j \in J\right\}$ be a pre open soft cover of $\tilde{X}$. Since the soft closure of each pre open soft set is semi open soft. Then $\left\{c l\left(F_{j}, E\right): j \in J\right\}$ is a cover of semi open soft sets of $\tilde{X}$. By hypothesis, there exists finite $J_{o} \subseteq J$ such that $\tilde{X}-\tilde{\cup}\left\{\left(F_{j}, E\right): j \in J_{o}\right\} \in \tilde{I}$.

Corollary 3.2. If $(X, \tau, E, \tilde{I})$ is soft semi- $\tilde{I}$-compact, then any pre closed family $\left\{\left(F_{j}, E\right): j \in J\right\}$ for which $\tilde{\cap}\left\{\left(F_{j}, E\right): j \in J\right\}=\tilde{\phi}$, there exists finite $J_{o} \subseteq J$ such that $\tilde{\cap}\left\{\operatorname{int}\left(F_{j}, E\right): j \in J_{o}\right\} \in \tilde{I}$.

Proof. Let $\left\{\left(F_{j}, E\right): j \in J\right\}$ be a family of pre closed soft subsets of $\tilde{X}$ such that $\tilde{\cap}\left\{\left(F_{j}, E\right): j \in J\right\}=\tilde{\phi}$. Then $\tilde{\cup}\left\{\tilde{X}-\left(F_{j}, E\right): j \in J\right\}$ is a family of pre open soft sets of $\tilde{X}$. Then there exists finite $J_{O} \subseteq J$ such that $\tilde{X}-\tilde{\cup}\left\{c l\left(\tilde{X}-\left(F_{j}, E\right)\right): j \in J_{o}\right\}=\tilde{\cap}\left\{\operatorname{int}\left(F_{j}, E\right): j \in J_{o}\right\} \in$ $\tilde{I}$ from Theorem 3.15.

\section{Soft countably semi- $\tilde{I}$-compactness spaces}

Definition 4.1. A soft subset $(F, E)$ of the space $(X, \tau, E, \tilde{I})$ is said to be soft countably- $\tilde{I}$-compact if and only if for every countable soft cover $\left\{\left(G_{\alpha}, E\right): \alpha \in \Lambda\right\}$ of $(F, E)$ by open soft sets, there exists a finite subset $\Lambda_{o}$ of $\Lambda$ such that $(F, E)-\tilde{\cup}_{\alpha \in \Lambda_{o}}\left(G_{\alpha}, E\right) \in \tilde{I}$.

The space $(X, \tau, E, \tilde{I})$ is said to be soft countably- $\tilde{I}$-compact if $\tilde{X}$ is soft countably $\tilde{I}$-compact as a soft subset.

Definition 4.2. A soft subset $(F, E)$ of the space $(X, \tau, E, \tilde{I})$ is said to be soft countably semi- $\tilde{I}$-compact if and only if 
for every countable soft cover $\left\{\left(G_{\alpha}, E\right): \alpha \in \Lambda\right\}$ of $(F, E)$ by semi open soft sets, there exists a finite subset $\Lambda_{o}$ of $\Lambda$ such that $(F, E)-\tilde{\cup}_{\alpha \in \Lambda_{o}}\left(G_{\alpha}, E\right) \in \tilde{I}$.

The space $(X, \tau, E, \tilde{I})$ is said to be soft countably semi- $\tilde{I}$ compact if $\tilde{X}$ is soft countably semi- $\tilde{I}$-compact as a soft subset.

From the previous Definition we have the following proposition.

\section{Proposition 4.1.}

(1)A space $(X, \tau, E, \tilde{I})$ is soft countably semi- $\langle\tilde{\phi}\rangle$-compact if and only if it is soft semi lindelöf.

(2)Every soft semi- $\tilde{I}$-compact space is soft countably semi- $\tilde{I}$-compact.

(3)A space $(X, \tau, E)$ is soft semi lindelöf if and only if it is soft countably semi- $\tilde{I}_{f}$-compact.

(4)Every soft countably semi- $\tilde{I}$-compact space is soft countably- $\tilde{I}$-compact.

Theorem 4.1. Let $(X, \tau, E, \tilde{I})$ be a soft countably semi- $\tilde{I}$ compact soft topological space and $\tilde{J}$ be a soft ideal on $X$ with the same set of parameters $E$ such that $\tilde{I} \subseteq \tilde{J}$. Then $(X, \tau, E, \tilde{J})$ is a soft countably semi- $\tilde{J}$-compact.

Proof. Immediate.

Theorem 4.2. If $(X, \tau, E, \tilde{I})$ is a soft countably semi- $\tilde{I}$-compact and soft semi lindelöf, then $(X, \tau, E, \tilde{I})$ is a soft semi- $\tilde{I}$-compact.

Proof. Let $\left\{\left(F_{j}, E\right): j \in J\right\}$ be a semi open soft cover of $\tilde{X}$. Since $\tilde{X}$ is soft semi lindelöf, then there exists a finite soft countable subcover $\left\{\left(F_{j}, E\right): j \in J_{o}\right\}$ such that $\tilde{X}=\tilde{\cup}_{j \in J_{o}}\left(F_{j}, E\right)$. Since $\tilde{X}$ is soft countably semi- $\tilde{I}$-compact, then $(X, \tau, E, \tilde{I})$ is soft semi- $\tilde{I}$-compact.

Theorem 4.3. Let $(X, \tau, E, \tilde{I})$ be a soft topological space with soft ideal. Then the following are equivalent:

(1) $(X, \tau, E)$ is soft countably semi- $\tilde{I}$-compact.

(2)For every countably family $\left\{\left(F_{j}, E\right): j \in J\right\}$ of semi closed soft subsets of $\tilde{X}$ for which $\tilde{\cap}\left\{\left(F_{j}, E\right): j \in J\right\}=$ $\tilde{\phi}$, there exists finite $J_{o} \subseteq J$ such that $\tilde{\cap}\left\{\left(F_{j}, E\right): j \in\right.$ $\left.J_{o}\right\} \in \tilde{I}$.

Proof. It is similar to the proof of Theorem 3.13.

Theorem 4.4.If $(X, \tau, E)$ is soft countably semi-I-I-compact, then for every countable family $\left\{\left(F_{j}, E\right): j \in J\right\}$ of regular closed soft subsets of $\tilde{X}$ such that $\tilde{\cap}\left\{\left(F_{j}, E\right): j \in J\right\}=\tilde{\phi}$, there exists finite $J_{o} \subseteq J$ such that $\tilde{X}-\tilde{\cup}\left\{\left(F_{j}, E\right): j \in J_{o}\right\} \in \tilde{I}$.

Proof. It is similar to the proof of Theorem 3.14.

Theorem 4.5. Let $\left(X_{1}, \tau_{1}, A, \tilde{I}\right)$ be a soft topological space with soft ideal, $\left(X_{2}, \tau_{2}, B\right)$ be a soft topological space and $f_{p u}:\left(X_{1}, \tau_{1}, A, \tilde{I}\right) \rightarrow\left(X_{2}, \tau_{2}, B\right)$ be an irresolute surjective soft function. If $\left(X_{1}, \tau_{1}, A\right)$ is soft countably semi- $\tilde{I}$-compact, then $\left(X_{2}, \tau_{2}, B\right)$ is soft countably semi- $f_{p u}(\tilde{I})$-compact.

Proof.

It is similar to the proof of Theorem 3.10.

Theorem 4.6. Let $\left(X_{1}, \tau_{1}, A\right)$ be a soft topological space, $\left(X_{2}, \tau_{2}, B, \widetilde{J}\right)$ be a soft topological space with soft ideal and $f_{p u}:\left(X_{1}, \tau_{1}, A\right) \rightarrow\left(X_{2}, \tau_{2}, B, \widetilde{J}\right)$ be a bijection irresolute open soft function. If $\left(X_{2}, \tau_{2}, B\right)$ is soft countably semi- $\tilde{J}$ compact, then $\left(X_{1}, \tau_{1}, A\right)$ is soft countably semi- $f_{p u}^{-1}(\tilde{J})$ compact.

\section{Proof.}

It is similar to the proof of Theorem 3.12.

\section{Conclusion}

Topology is an important and major area of mathematics and it can give many relationships between other scientific areas and mathematical models. Recently, many scientists have studied the soft set theory, which is initiated by Molodtsov and easily applied to many problems having uncertainties from social life. In the present work, we have continued to study the properties of soft topological spaces. we introduce the idea of new types of soft compactness defined in terms of soft ideal $\tilde{I}$ in a soft topological space $(X, \tau, E)$ namely, soft $\tilde{I}$-compactness, soft semi- $\tilde{I}$-compactness, soft countably $\tilde{I}$-compactness and soft countably semi-I-I-compactness. Also, several of their topological properties are investigated. Finally, some effects of various kinds of soft functions on them are studied. and have established several interesting properties. Because there exists compact connections between soft sets and information systems $[26,30]$, we can use the results deducted from the studies on soft topological space to improve these kinds of connections. We see that this paper will help researcher enhance and promote the further study on soft topology to carry out a general framework for their applications in practical life.

\section{References}

[1] M. E. Abd El-Monsef, E. F. Lashien and A. A. Nasef, Scompactness via ideals, Qatar univ. Sci. J., 11, 33-44 (1991).

[2] B. Ahmad and A. Kharal, On fuzzy soft sets, Advances in Fuzzy Systems, 1-6 (2009).

[3] B. Ahmad and A. Kharal, Mappings of soft classes, to appear in New Math. Nat. Comput. 
[4] H. Aktas and N. Cagman, Soft sets and soft groups, Information Sciences, 1, 2726-2735 (2007).

[5] M. I. Ali, F. Feng, X. Liu, W. K. Min and M. Shabir, On some new operations in soft set theory, Computers and Mathematics with Applications, 57, 1547-1553 (2009).

[6] A. Aygunoglu and H. Aygun, Introduction to fuzzy soft groups, Computers and Mathematics with Applications, 58, 1279-1286 (2009).

[7] Banu Pazar Varol and Halis Aygun, On soft Hausdorff spaces, Annals of Fuzzy Mathematics and Informatics, 5, 15-24 (2013).

[8] N. agman, F.itak and S. Enginoglu, Fuzzy parameterized fuzzy soft set theory and its applications, Turkish Journal of Fuzzy Systems, 1, 21-35 (2010).

[9] N.agman and S. Enginoglu, Soft set theory and uniint decision making, European Journal of Operational Research, 207, 848-855 (2010).

[10] D. Jankovic and T.R. Hamlet, New topologies from old via ideals, The American Mathematical Monthly, 97, 295-310 (1990).

[11] A.Kandil, O. A. E. Tantawy, S. A. El-Sheikh and A. M. Abd El-latif, $\gamma$-operation and decompositions of some forms of soft continuity in soft topological spaces, Annals of Fuzzy Mathematics and Informatics, 7, 181-196 (2014).

[12] A.Kandil, O. A. E. Tantawy, S. A. El-Sheikh and A. M. Abd El-latif, Soft ideal theory, Soft local function and generated soft topological spaces. To appear in the journal Applied Mathematics $\propto$ Information Sciences.

[13] E.F. Lashina, A.M. Kozae, A.A. Abo Khadra, T. Medhat, Rough set theory for topological spaces, International Journal of Approximate Reasoning, 40, 35-43 (2005).

[14] A.Kandil, O. A. E. Tantawy, S. A. El-Sheikh and A. M. Abd El-latif, Supra soft topological spaces. To appear in the Jökull Journal.

[15] K. Kannan, Soft generalized closed sets in soft topological spaces, Journal of Theoretical and Applied Technology, 37, 17-21 (2012)

[16] D. V. Kovkov, V. M. Kolbanov and D. A. Molodtsov, Soft sets theory-based optimization, Journal of Computer and Systems Sciences International, 46, 872-880 (2007).

[17] J. Mahanta and P.K. Das, On soft topological space via semi open and semi closed soft sets,arXiv:1203.4133v1, (2012).

[18] P. K. Maji, R. Biswas and A. R. Roy, Fuzzy soft sets, Journal of Fuzzy Mathematics, 9, 589-602 (2001).

[19] P. K. Maji, R. Biswas and A. R. Roy, Intuitionistic fuzzy soft sets, Journal of Fuzzy Mathematics, 9, 677-691 (2001).

[20] P. K. Maji,R. Biswas and A. R. Roy, Soft set theory, Computers and Mathematics with Applications, 45, 555-562 (2003).

[21] P. Majumdar and S. K. Samanta, Generalised fuzzy soft sets, Computers and Mathematics with Applications, 59, 14251432 (2010).

[22] D. A. Molodtsov, Soft set theory-firs tresults, Computers and Mathematics with Applications, 37, 19-31 (1999).
[23] D.Molodtsov, V. Y. Leonov and D. V. Kovkov, Soft sets technique and its application, Nechetkie Sistemy i Myagkie Vychisleniya, 1, 8-39 (2006) .

[24] A. Mukherjee and S. B. Chakraborty, On intuitionistic fuzzy soft relations, Bulletin of Kerala Mathematics Association, 5, 35-42 (2008).

[25] Sk. Nazmul and S. K. Samanta, Neighbourhood properties of soft topological spaces, Annals of Fuzzy Mathematics and Informatics, 6, 1-15 (2012).

[26] D. Pei and D. Miao, From soft sets to information systems, in: X. Hu, Q. Liu, A. Skowron, T. Y. Lin, R. R. Yager, B. Zhang (Eds.), Proceedings of Granular Computing, in: IEEE, 2, 617-621 (2005).

[27] S. Hussain and B. Ahmad, Some properties of soft topological spaces, Comput. Math. Appl., 62, 4058-4067 (2011).

[28] M. Shabir and M. Naz, On soft topological spaces, Comput. Math. Appl., 61, 1786-1799 (2011).

[29] Sujoy Das and S. K. Samanta, Soft metric, Annals of Fuzzy Mathematics and Informatics, 6, 77-94 (2013).

[30] Z. Xiao, L. Chen, B. Zhong, S. Ye, Recognition for information based on the theory of soft sets, in: J. Chen(Ed.), Proceeding of ICSSSM-05, IEEE, 2, 1104-1106 (2005).

[31] Weijian Rong, The countabilities of soft topological spaces, International Journal of Computational and Mathematical Sciences, 6, 159-162 (2012).

[32] W. Xu, J. Ma, S. Wang and G. Hao, Vague soft sets and their properties, Computers and Mathematics with Applications, 59, 787-794 (2010).

[33] Y. Zou and Z. Xiao, Data analysis approaches of soft sets under incomplete information, Knowledge-Based Systems, 21, 941-945 (2008).

[34] I. Zorlutuna, M. Akdag, W.K. Min and S. Atmaca, Remarks on soft topological spaces, Annals of Fuzzy Mathematics and Informatics, 3, 171-185 (2012).

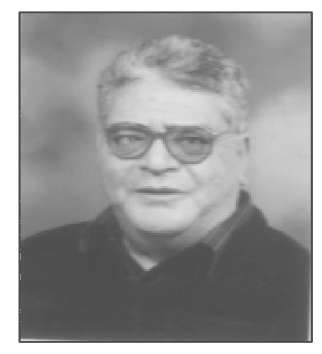

A. K. S. Ibrahim is a Professor of Mathematics at Helwan University. He received the Ph.D. degree in Topology from the University of Moscow in 1978. His primary research areas are General Topology, Fuzzy Topology, double sets and theory of sets. Dr. Kandil has published over 80 papers in refereed journals and contributed several book chapters in various types of Mathematics textbooks. He is a Fellow of the Egyptian Mathematical Society and Egyptian Physics Mathematical Society. He was the Supervisor of 20 PHD and about 30 MSC students. 


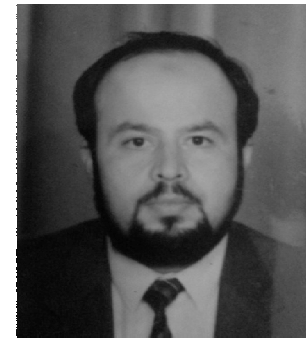

O. A. El-Tantawy is a Professor of Mathematics at Zagazig University. He born in 1951. He received the Ph.D. degree in Topology from the University of Zagazig in 1988. His primary research areas are General Topology, Fuzzy Topology, double sets and theory of sets. Dr. Osama has published over 50 papers in refereed journals. He is a Fellow of the Egyptian Mathematical Society and Egyptian Physics Mathematical Society. He was the Supervisor of 10 PHD and about 17 MSC students.

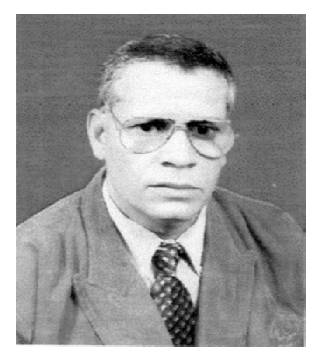

S. A. A. El-Sheikh is an assistance Professor of pure Mathematics, Ain Shams University ,Faculty of Education, Mathematic Department, Cairo, Egypt. He born in 1955. He received the $\mathrm{Ph} . \mathrm{D}$. degree in Topology from the University of Zagazig. His primary research areas are General Topology, Fuzzy Topology, double sets and theory of sets. Dr. Sobhy has published over 15 papers in Fuzzy set and system Journal (FSS), Information science Journal (INFS), Journal of fuzzy Mathematics and Egyptian Journal of Mathematical Society. He was the Supervisor of many PHD MSC Thesis.

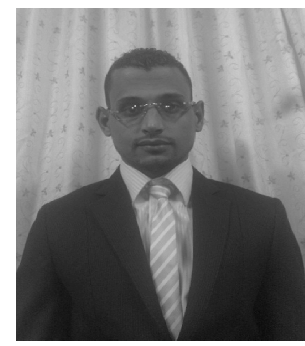

A. M. A. Daby is a Ph.D student in pure Mathematics (Topology) Ain Shams University ,Faculty of Education, Mathematic Department, Cairo, Egypt. He was born in 1985. He received the MSC Thesis degree in Topology from Ain Shams University in 2012. His primary research areas are General Topology, Fuzzy Topology, Set theory, Soft set theory and Soft topology. Dr. Alaa has published many papers in refereed journals. 